\title{
Characterization of pandemic influenza immune memory signature after vaccination or infection
}

\author{
Olivia Bonduelle,1,2,3 Fabrice Carrat, ${ }^{3,4,5,6}$ Charles-Edouard Luyt,,3,7 Catherine Leport, ${ }^{3,8,9,10}$ \\ Anne Mosnier, ${ }^{3,11}$ Nora Benhabiles, ${ }^{3,12}$ Anne Krivine,,3,13 Flore Rozenberg, ${ }^{3,13}$ Nora Yahia,, ${ }^{1,2,3}$ \\ Assia Samri,,,2,3 Dominique Rousset,3,14,15 Sylvie van der Werf,3,15 \\ Brigitte Autran, 1,2,3,16 and Behazine Combadiere ${ }^{1,2,3}$
}

\begin{abstract}
${ }^{1}$ Sorbonne Universités, UPMC University Paris 06, UMR S CR7, Centre d'Immunologie et de Maladies Infectieuses - Paris (Cimi-Paris), Paris, France. 2INSERM U1135, Cimi-Paris, Paris, France. ${ }^{3}$ Memo-Flu-ARDS Study Group. ${ }^{4}$ Sorbonne Universités, UPMC University Paris 06, UMR S 1136, Institut Pierre Louis d'Epidémiologie et de Santé Publique (iPLESP), Paris, France. 5INSERM U1136, iPLESP, Paris, France. ${ }^{6}$ Département de Santé Publique, Hôpital Saint-Antoine, Assistance Publique/Hôpitaux de Paris (AP/HP), Paris, France. ${ }^{7}$ Service de Réanimation Médicale, Institut de Cardiologie, AP/HP, Paris, France. ${ }^{8}$ AME (Infection, Antimicrobials, Modelisation, Evolution), UMR 1137, University Paris Diderot, Sorbonne Paris Cité,

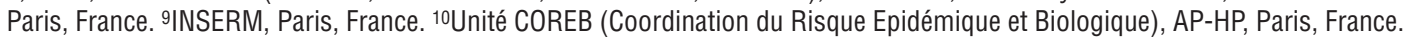

${ }^{11}$ Coordination Nationale du Réseau des Groupes Régionaux d’Observation de la Grippe (GROG), Paris, France.

${ }^{12}$ Commissariat à l'Energie Atomique et aux Energies Alternatives (CEA) Saclay, Institut Carnot LIST Department Sensors Signal and Information, Gif-sur-Yvette, France. ${ }^{13}$ Université Paris Descartes EA1833 et Service de Virologie, Hôpital Cochin, Paris, France. ${ }^{14}$ Institut Pasteur de la Guyane, Cayenne, Guyane, France. ${ }^{15}$ Institut Pasteur, Unité de Génétique Moléculaire des Virus à ARN, CNR des Virus Influenzae,

Département de Virologie and CNRS UMR3569, Université Paris Diderot, Sorbonne Paris Cité, Paris, France. 16Département d’Immunologie,
\end{abstract} Hôpital Pitié-Salpêtrière, AP/HP, Paris, France.

\begin{abstract}
The magnitude, quality, and maintenance of immunological memory after infection or vaccination must be considered for future design of effective influenza vaccines. In 2009, the influenza pandemic produced disease that ranged from mild to severe, even fatal, illness in infected healthy adults and led to vaccination of a portion of the population with the adjuvanted, inactivated influenza $A(H 1 N 1) p d m 09$ vaccine. Here, we have proposed a multiparameter quantitative and qualitative approach to comparing adaptive immune memory to influenza 1 year after mild or severe infection or vaccination. One year after antigen encounter, severely ill subjects maintained high levels of humoral and polyfunctional effector/memory $\mathrm{CD}^{+} \mathrm{T}$ cells responses, while mildly ill and vaccinated subjects retained strong cellular immunity, as indicated by high levels of mucosal homing and degranulation markers on IFN- $\gamma^{+}$antigen-specific T cells. A principal component analysis distinguished 3 distinct clusters of individuals. The first group comprised vaccinated and mildly ill subjects, while clusters 2 and 3 included mainly infected individuals. Each cluster had immune memory profiles that differed in magnitude and quality. These data provide evidence that there are substantial similarities between the antiinfluenza response that mildly ill and vaccinated individuals develop and that this immune memory signature is different from that seen in severely ill individuals.
\end{abstract}

\section{Introduction}

Influenza A infection is still a major infectious disease that has appreciable morbidity and mortality during the annual seasonal epidemic as during the 2009 pandemic (1). Genetic mutations are typically responsible for seasonal influenza outbreaks (2), and the emergence of an influenza pandemic results from genetic reassortment among different strains and subtypes (3). Ab responses acquired after natural infection or seasonal vaccination do not confer long-time protection against all strains because of the high seasonal-virus variability (4). In addition, cellular immunity recognizes conserved regions of influenza proteins $(5,6)$ and is reported to play a role in reducing morbidity from influenza infection (7). Here, we examined and compared the level of persistence of antibody and cellular memory responses both to influenza A(H1N1)pdm09 vaccination and to pandemic infection, either mild or severe.

Conflict of interest: Fabrice Carrat has received consulting fees from GlaxoSmith Kline. Charles-Edouard Luyt has received consulting fees from Bayer and lecture fees from Thermo Fisher Scientific Brahms and GlaxoSmithKline. Behazine Combadiere has received consulting fees from Sanofi Pasteur MSD, Pfizer, and Merck. Sylvie van der Werf has received consulting fees from Danone through her research unit. Citation for this article: J Clin Invest. 2014;124(7):3129-3136. doi:10.1172/JCI74565
Pandemic influenza viruses often cause severe disease in young or middle-aged adults, even those without preexisting comorbidities (8-10). The mechanism of illness associated with severe disease in this age group is not well understood (11), but might involve impaired immune responses. Serum Abs that crossreact with, but do not protect against, influenza $\mathrm{A}(\mathrm{H} 1 \mathrm{~N} 1) \mathrm{pdm} 09$ virus can be found in middle-aged but not in young adults. These nonprotective Abs have been shown to induce immune complex-mediated disease after infection (12). High titers of serum Abs of low avidity for H1-2009 antigen in severely ill individuals have often been associated with mortality (12). To et al., however, have proposed that nonneutralizing Abs with high avidity might also be associated with severe illness during influenza A(H1N1)pdm09 infection (13, 14). Individuals who survive are thus those who mounted an immune response.

The magnitude and functional characteristics of immune memory involving both $\mathrm{Ab}$ and $\mathrm{T}$ cell responses are key parameters of protection against future infections. As reported by Hobson et al. in 1972 (15), neutralizing $\mathrm{Ab}$ response is the reference criterion for evaluating the efficacy of influenza immunity for potential protection after vaccination. Other aspects of immunity are also relevant, however, such as serum avidity and $\mathrm{T}$ cell responses, and 
their polyfunctionality has been associated with protection (16, 17). Indeed, influenza-specific $T$ cells play a crucial role in the control of viral influenza infection and are capable of producing cytokines and killing infected cells $(7,18)$. Still other aspects to be considered are postinfection mucosal and cell trafficking (19).

The mechanisms that induce these characteristics depend on 2 parameters that differ substantially according to whether vaccination or infection-induced immunization has occurred: antigen dose and duration of exposure. In the absence of circulating antigen, immune memory is considered to have reached a resting state a year after the first encounter (20). It is not yet clear whether vaccination generates levels and characteristics of immune memory similar to those induced by infection itself, although adjuvants to vaccines are supposed to enhance both the signal strength and exposure duration. We recently proposed that the extreme heterogeneity of immune responses to influenza $\mathrm{A}(\mathrm{H} 1 \mathrm{~N} 1) \mathrm{pmd} 09$ three weeks after vaccination is related to individual factors, including the magnitude and quality of influenza-specific immune responses before vaccination (21).

We thus examined the immunologic status of cellular and humoral memory of influenza A(H1N1)pdm09 to define and compare the immune memory signature of severe and mild infection and of adjuvanted vaccination. We explored the plexus of immune parameters that might be used as markers of protection against influenza viruses. We found some significant similarities between mildly ill and vaccinated subjects, who shared an immune memory signature different from the two seen in severely ill individuals.

\section{Results}

The intensity of effector/memory bumoral and cellular immune responses distinguishes individuals who had severe influenza A(H1N1)pdm09 infections from mildly ill or vaccinated subjects 1 year later. One year after influenza $\mathrm{A}(\mathrm{H} 1 \mathrm{~N} 1) \mathrm{pdm} 09$ vaccination or infection, we studied immunologic memory parameters reported to play a role in either protection against influenza $\mathrm{A}(\mathrm{H} 1 \mathrm{~N} 1)$ pdm09 infection or its severity. These parameters included hemagglutinin inhibition (HI) titers (Figure $1 \mathrm{~A}$ ), serum $\mathrm{Ab}$ avidity (Figure $1 \mathrm{~B}$ ), counts of $\mathrm{A}(\mathrm{H} 1 \mathrm{~N} 1)$ pdm09 virus-specific IFN- $\gamma / \mathrm{IL}-2 / \mathrm{TNF}-\alpha-$-secreting $\mathrm{CD}^{+}$ and $\mathrm{CD}^{+} \mathrm{T}$ cells (Figure 1, C and D, respectively), degranulation capacity with $\mathrm{A}(\mathrm{H} 1 \mathrm{~N} 1)$ pdm09-specific $\mathrm{CD} 107 \mathrm{a}^{+} \mathrm{CD} 4^{+}$and $\mathrm{CD}^{+}$ $\mathrm{T}$ cells (Figure 1, E and F, respectively), and lung/mucosa-associated homing markers: CD49a $\mathrm{a}^{+} \mathrm{CD} 49 \mathrm{~d}^{+} \mathrm{CD} 4^{+}$effector $\mathrm{T}$ cells (Figure $1 \mathrm{G}$ ) and $\mathrm{CD} 49 \mathrm{a}^{+} \mathrm{CD} 49 \mathrm{~d}^{+} \mathrm{CD}^{+}$effector T cells (Figure $1 \mathrm{H}$ and ref. 19). Figure $1 \mathrm{I}$ is a radar chart summarizing adaptive memory immunity 1 year after infection or vaccination.

We found that the intensity of humoral responses (HI titers) as well as of $\mathrm{A}(\mathrm{H} 1 \mathrm{~N} 1)$ pdm09-specific cytokine-secreting $\mathrm{CD}^{+}$ effector/memory $\mathrm{T}$ cells was significantly higher at 1 year after a severe $\mathrm{A}(\mathrm{H} 1 \mathrm{~N} 1) \mathrm{pdm} 09$ infection than after vaccination; a similar trend existed when we compared severe to mild infection (HI titers: severe compared with vaccination: $P=0.0474$ and compared with mild: $P=0.0544$; $C D 4^{+} \mathrm{T}$ cells: severe compared with vaccination: $P=0.0272$ and compared with mild: $P=0.0038)$. On the other hand, the intensity of the cellular response, including IFN $-\gamma^{+} \mathrm{CD} 4^{+}$ or $\mathrm{CD}^{+} \mathrm{T}$ cells bearing mucosa homing markers (CD49a and CD49d), was greater in mildly infected or vaccinated subjects than those severely ill a year earlier $(P<0.05)$. Expression of the degranulation marker (CD107a) on effector/memory CD4 ${ }^{+}$and $\mathrm{CD}^{+}$ $\mathrm{T}$ cells was also significantly more intense during the memory phase after vaccination, reflecting cellular response with cytotoxic potential $(P<0.01)$. It should be noted that serum avidity and the frequency of cytokine-producing $\mathrm{CD}^{+} \mathrm{T}$ cells specific for influenza $\mathrm{A}(\mathrm{H} 1 \mathrm{~N} 1)$ pdm09 remained similar in all groups.

These results show distinct imprinting by infection and vaccination at 1 year after severe and mild infection and vaccination. To summarize these similarities and disparities, we examined the intensity of 8 parameters among the groups. In individuals with mild infection compared with vaccinated subjects, the intensity of $6 / 8$ parameters was similar (HI titers, serum avidity, mucosa-homing effector $\mathrm{CD}^{+}$ and $\mathrm{CD}^{+} \mathrm{T}$ cells, and cytokine-secreting effector/memory $\mathrm{CD}^{+}$and $\mathrm{CD}^{+} \mathrm{T}$ cells); in those with severe and mild infections, the intensity of $5 / 8$ was similar (HI titers, serum avidity, cytokine-secreting CD8 ${ }^{+}$ T cells and CD107a expression on effector/memory CD4 $4^{+}$and $\mathrm{CD}^{+}$ $\mathrm{T}$ cells); and in those with severe infection and those vaccinated, the intensity of only $2 / 8$ was similar (serum avidity and effector/ memory $\mathrm{CD}^{+} \mathrm{T}$ cells). Overall, the immune memory signature of severely ill individuals tended toward a high level of HI titers and cytokine-producing effector/memory $\mathrm{CD}^{+} \mathrm{T}$ cells, whereas mildly ill and vaccinated subjects, despite significant $\mathrm{HI}$ titers, had higher levels of mucosa-homing T cells and CD $107 \mathrm{a}^{+} \mathrm{T}$ cells in the blood at the memory phase than the severely ill subjects.

Distinct combinations of polyfunctionality of $C D 4^{+}$and $C D 8^{+} T$ cells determine memory responses for individuals who had severe influenza infections, but these were strongly similar for mildly ill and vaccinated subjects. One hallmark of the efficacy of $\mathrm{T}$ cell responses against viral infection is the production of multiple cytokines by $\mathrm{CD}^{+}$ and $\mathrm{CD}^{+}$cells. Figure $1, \mathrm{C}$ and D, presents data according to the total frequency of triple-positive (TP) $\left(\right.$ IFN- $\gamma^{+}$IL-2+ TNF- $\left.\alpha^{+}\right)$, double-positive (DP) (IFN- $\gamma^{+}$IL-2 ${ }^{+}$TNF- $\alpha^{-}$, IFN- $\gamma^{+}$IL-2-TNF- $\alpha^{+}$or IFN- $\gamma^{-}$IL-2 ${ }^{+}$TNF- $\left.\alpha^{+}\right)$, and single-positive (SP) (IFN- $\gamma^{+}$IL-2-TNF- $\alpha^{-}$, IFN- $\gamma^{-}$IL-2 ${ }^{+}$TNF- $\alpha^{-}$or IFN- $\gamma^{-}$IL-2-TNF- $\left.\alpha^{+}\right)$cytokine-secreting $\mathrm{T}$ cells. Figure 2, A and C, depict the percentages of A(H1N1) pdm09-specific TP, DP, and SP cytokine-secreting $\mathrm{CD}^{+}$and $\mathrm{CD}^{+} \mathrm{T}$ cells found 1 year after severe or mild infection or vaccination. The results are summarized in separate radar charts for $\mathrm{CD}^{+}$and $\mathrm{CD}^{+} \mathrm{T}$ cells (Figure 2, B and D).

In total $\mathrm{A}(\mathrm{H} 1 \mathrm{~N} 1) \mathrm{pdm} 09$-specific memory $\mathrm{CD}^{+} \mathrm{T}$ cells, severe influenza infection induced higher amplification of SP, DP, and TP cytokine-secreting memory $\mathrm{CD}^{+}$cells, but only the TP, IFN- $\gamma^{-}$ IL- ${ }^{+}$TNF- $\alpha^{+}$and IFN- $\gamma^{-}$IL-2-TNF- $\alpha^{+}$subpopulations differed significantly between severe and mild ill or vaccinated subjects $(P<0.01$; Figure 2, A and B). No differences were observed when comparing vaccination to mild infection except for SP IFN- $\gamma^{+}$IL-2TNF- $\alpha^{-}$-producing $\mathrm{CD}^{+} \mathrm{T}$ cells, which were more numerous at the memory phase after vaccination $(P=0.0134$; Figure 2 , A and $\mathrm{B})$.

The $\mathrm{CD}^{+}$cytokine profile, however, did not differ significantly among groups (Figure 2, C and D); although the proportion of most cytokine-producing effector/memory $\mathrm{CD}^{+} \mathrm{T}$ cells tended to be higher after severe infection, the difference was statistically insignificant. Some discrepancies were observed between memory responses after mild infection compared with vaccination; in the former, we found a higher percentage of IFN- $\gamma^{+}$IL-2-TNF- $\alpha^{-}$and in the latter, a higher percentage of IFN- $\gamma^{-}$IL-2-TNF- $\alpha^{+}$.

Principal component analyses revealed distinct clusters of individuals with similar immune memory signatures 1 year after the A(H1N1)pdm09 antigen encounter. To extract all the relevant information about all of the immune parameters amplified after vaccination or by severe or mild A(H1N1)pdm09 virus infection, we used a principal component analysis (PCA). This technique is an unsupervised visualization tool used to optimize our understanding of the underlying 
A

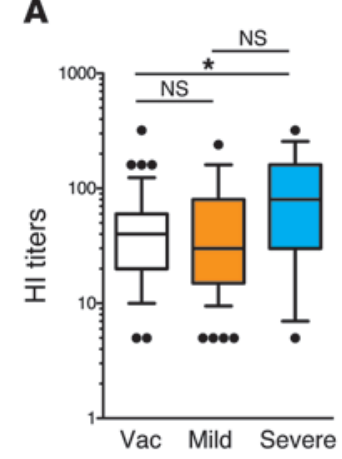

B
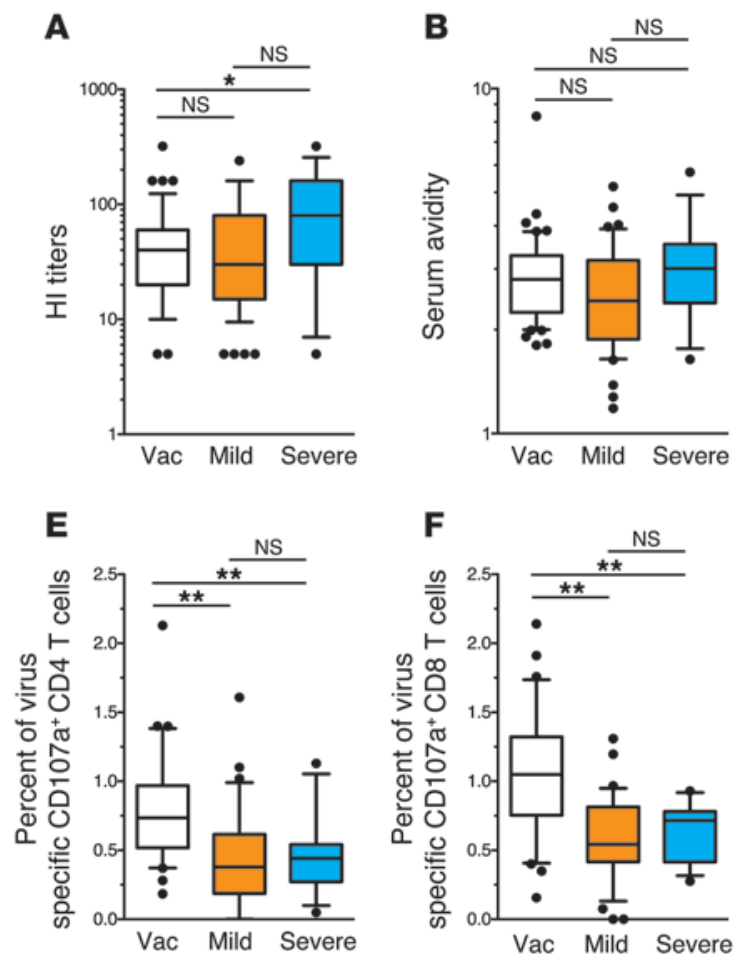

I

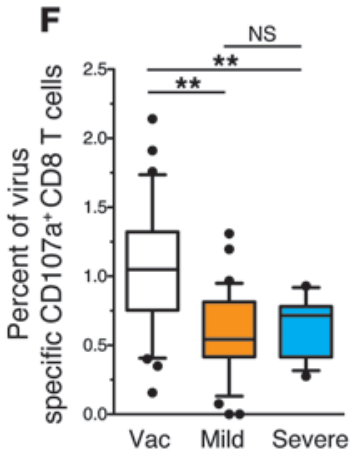

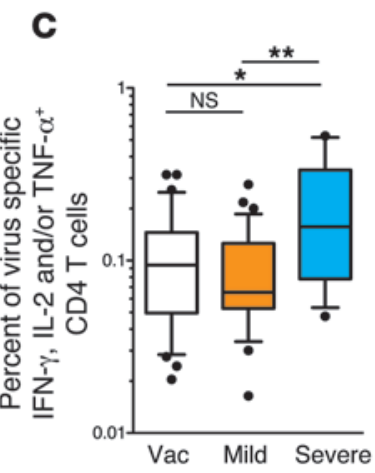
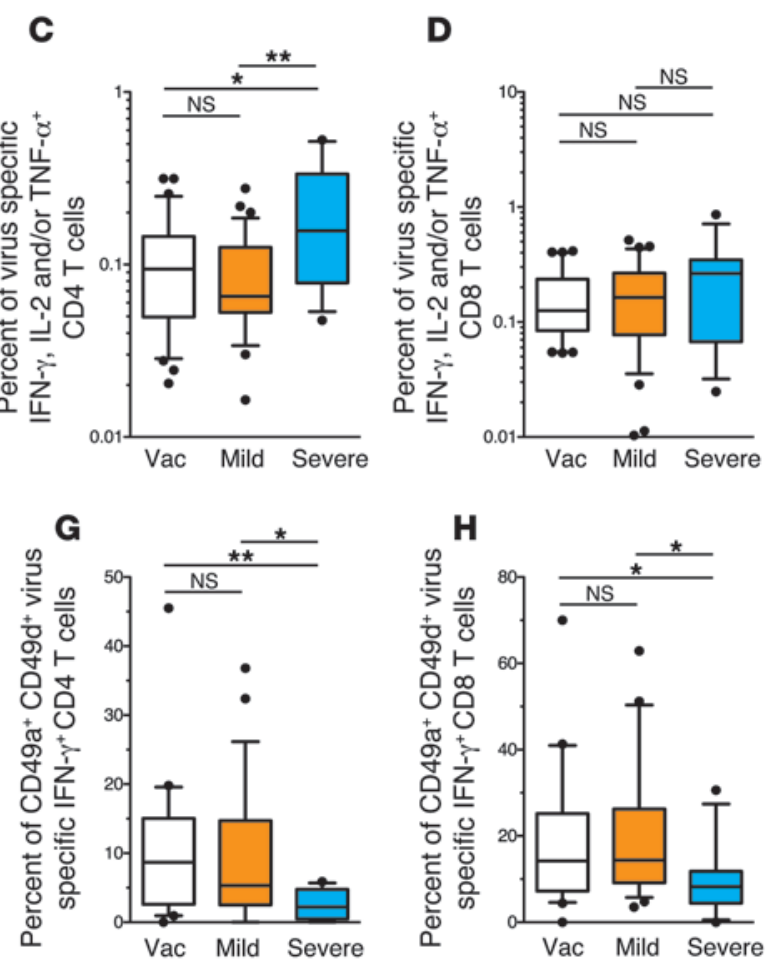

HI titers

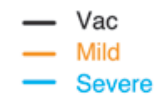

Serum avidity $\quad \ldots{ } \ldots . . . .{ }^{4}$

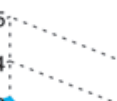

Virus-specific
CD49a+CD49d

- Severe

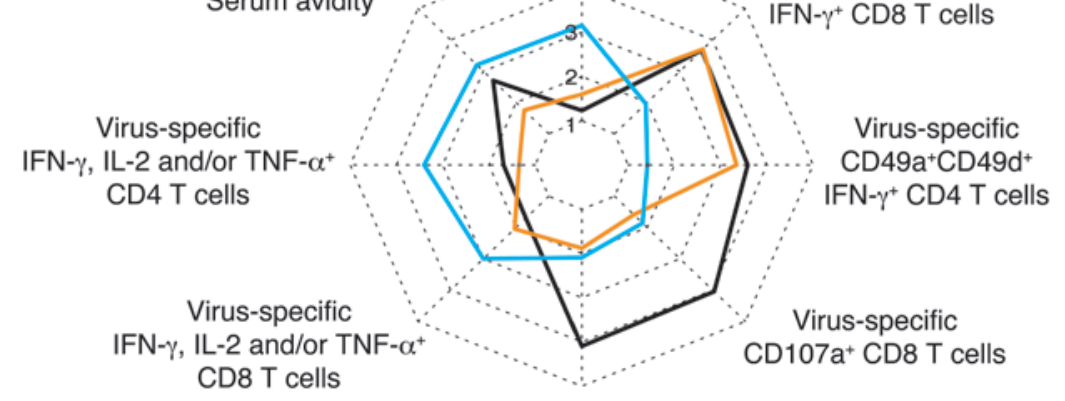

Virus-specific

CD107a+ CD4 T cells

\section{Figure 1}

Differential intensity of long-term immune response between vaccinated and infected subjects. A box-and-whiskers plot with the 10th to 90th percentiles is presented for each parameter. One year after the antigen encounter, we evaluated influenza-specific immune responses: (A) hemagglutination inhibition titers (HI titers) (HI titers $\geq 1 / 40$ : vaccination: 26/48 [54.17\%]; mild: $23 / 48$ [47.92\%]; severe: 10/13 [76.92\%]) and (B) serum avidity assay in 48 vaccinated subjects (white), 48 mildly ill subjects (orange), and 13 severely ill subjects (blue). The graph's $y$ axis is in $\log _{10}$ scale. (C and D) Influenza A(H1N1)pdm09 virus-specific CD4 ${ }^{+}$and $\mathrm{CD}^{+} \mathrm{T}$ cells were measured by secreted cytokines (Boolean gates for IFN- $\gamma$, IL-2, and/or TNF- $\alpha$ ) in 30 vaccinated, 32 mildly ill, and 13 severely ill subjects. The graph's $y$ axis is in log ${ }_{10}$ Scale. (E and F) Expression of influenza $\mathrm{A}\left(\mathrm{H} 1 \mathrm{N1}\right.$ )pdm09 virus-specific CD107a on $\mathrm{CD}^{+}$and $\mathrm{CD} 8^{+} \mathrm{T}$ cells was evaluated in samples from 30 vaccinated, 32 mildly ill, and 13 severely ill subjects; (G and $\mathbf{H})$ double CD49a- and CD49d-positive markers were evaluated on IFN- $\gamma^{+} \mathrm{CD} 4^{+}(\mathbf{G})$ and IFN- $\gamma^{+} \mathrm{CD} 8^{+}$ (H) T cells in samples from 20 vaccinated, 26 mildly ill, and 11 severely ill subjects. Statistical analyses were performed with the Mann Whitney $U$ test; statistical significance is indicated. ${ }^{*} P<0.05 ;{ }^{* *} P<0.01$. (I) Radar charts compare influenza virus-specific immune compartments of vaccinated (black), mildly ill (orange) and severely ill (blue) groups. The values on the axis represent the mean of each parameter derived from the upper and lower $95 \% \mathrm{Cls}$ of the mean of each assay for all tested subjects. vac, vaccinated volunteers, mild, patients with mild to moderate $A(H 1 N 1) p d m 09$ pandemic virus influenza infection; severe, patients with severe $A(H 1 N 1) p d m 09$ pandemic virus influenza infection.

structure of a data set (22). It enabled us to conduct an integrative analysis of the trends in the 8 immune parameters discussed above (HI titers, serum avidity, cytokine-producing $\mathrm{CD}^{+}$and $\mathrm{CD}^{+}$ $\mathrm{T}$ cells, CD107a+ $\mathrm{T}$ cells, and homing markers of effector $\mathrm{T}$ cells) after normalization. Figure $3 \mathrm{~A}$ presents the PCA. It clustered the 57 subjects for whom we had data for all 8 immune parameters into 3 sets that differed significantly. The first 3 components explain up to $83.28 \%$ of the variance. 
A

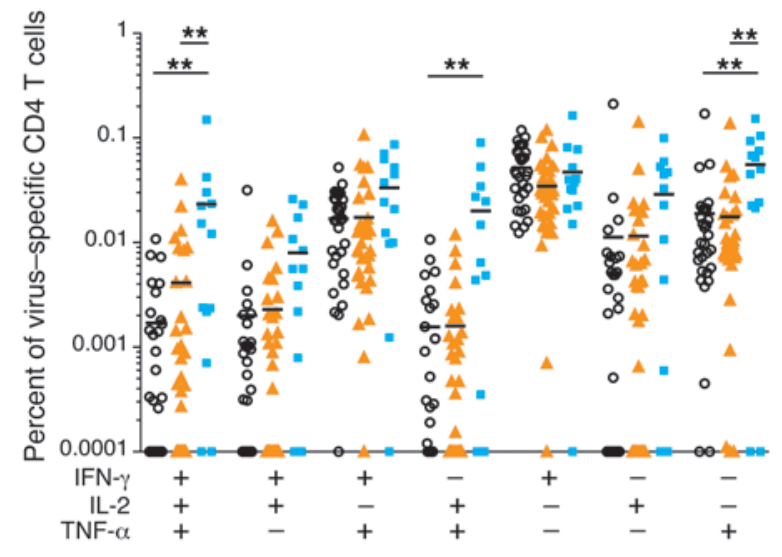

C

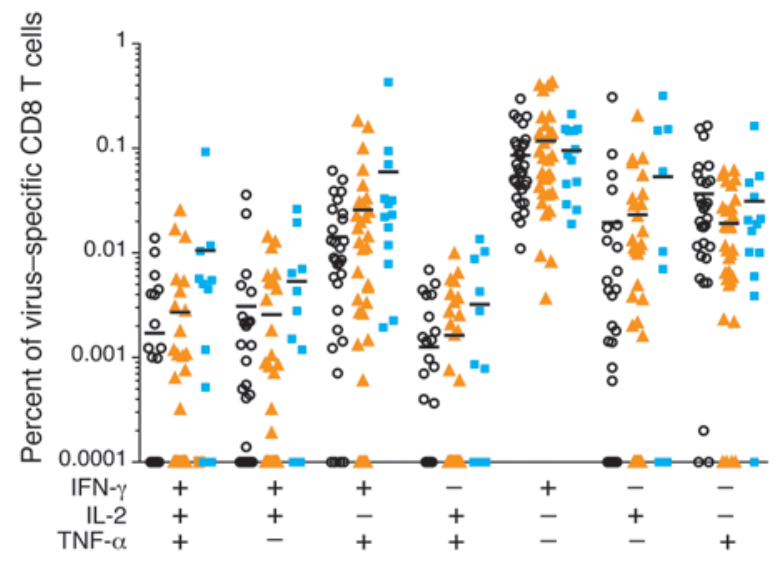

$\mathbf{B}$

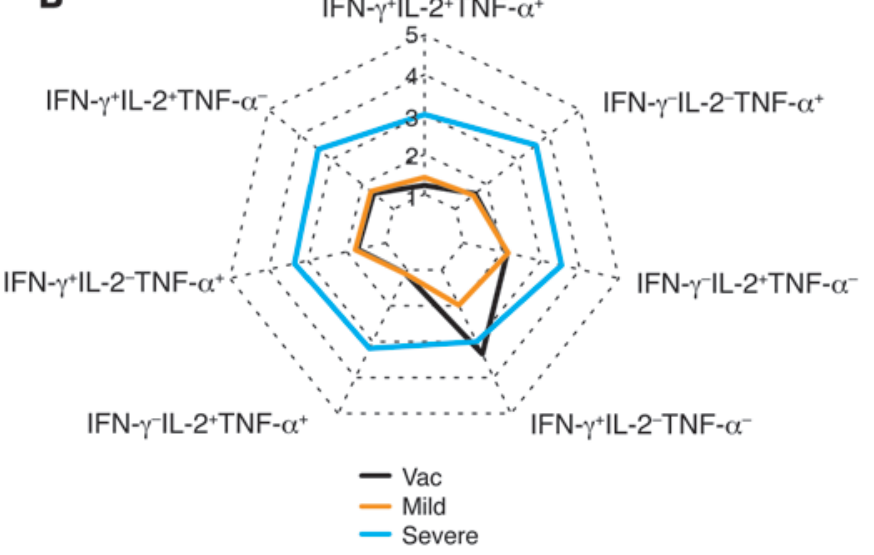

D

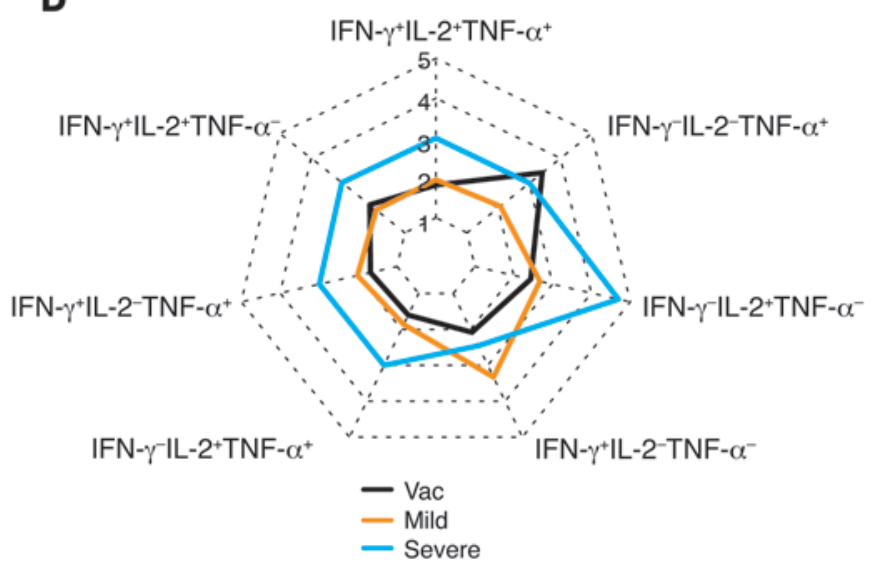

Figure 2

Polyfunctional influenza memory T cell responses were amplified after severe infection. Detailed analyses of SP, DP, and TP cytokine-producing $A(H 1 N 1) p d m 09$ virus-specific CD4+ $T$ cell responses $(\mathbf{A}$ and $\mathbf{B})$ and $C D 8^{+} T$ cell responses $(\mathbf{C}$ and $\mathbf{D})$ are shown on the $x$ axes for the vaccinated group ( $n=30$; white), the mildly ill group ( $n=32$; orange), and the severely ill group $(n=13$; blue). The graphs' $y$ axes is in log 10 scale. The means of each cytokine combination for each group are presented in black. Statistical analyses used the Mann-Whitney $U$ test and statistical significance is indicated. ${ }^{\star \star} P<0.01$. (B and D) Radar charts present the upper and lower $95 \% \mathrm{Cls}$ of the mean of each cytokine combination, as indicated in linear scale, for vaccinated (black), mildly ill (orange) and severely ill subjects (blue).

The first cluster (cluster $1, n=24$ ) contained mildly ill subjects $(n=11 ; 46 \%)$ and vaccinated subjects $(n=13 ; 54 \%)$; it underlines the similarities in the memory responses after vaccination and infection, as shown in Figure 3B. These results further confirm that the subjects who were mildly ill have memory responses similar to those of vaccinated subjects.

Cluster 2, which was much smaller $(n=8)$, and cluster 3 , which included 25 subjects, both comprised mainly infected individuals (Figure 3B), but they had 2 clearly different immune memory profiles. Cluster 2 included subjects with a lower intensity of immune memory but in which all immune functions were nonetheless represented. Cluster 3 , on the other hand, included subjects with average $\mathrm{HI}$ titers and homing $\mathrm{T}$ cells $\left(\mathrm{CD} 49 \mathrm{a}^{+} \mathrm{CD} 49 \mathrm{~d}^{+}\right)$. These results further underline some similarities at the memory phase between severely and mildly ill individuals.

\section{Discussion}

This work is the first study, to our knowledge, to propose an immune signature of the immunologic memory of an influenza
A(H1N1) virus based on 8 parameters of immunity, including humoral and cellular immune responses, in subjects at 1 year after A(H1N1)pdm09 vaccination or infection (severe or mild). At this memory phase, severely ill individuals could be distinguished by a significantly higher level of $\mathrm{HI}$ titers and $\mathrm{CD} 4^{+} \mathrm{T}$ cell polyfunctionality compared with mildly ill individuals and vaccinated individuals, whose results for these parameters could barely be told apart a year later.

As the world faced a new influenza pandemic in 2009, research focused on the persistence of neutralizing Abs against influenza strains that were genetically close to the influenza $\mathrm{A}(\mathrm{H} 1 \mathrm{~N} 1) \mathrm{pdm} 09$ strain, as imprinted during previous antigen encounters. The oldest cohort showed evidence of robust immunity to influenza A(H1N1)pdm09 with significant neutralizing capacities (23): subjects born before 1930 were probably exposed to 1918-like H1N1 and thus had high Ab titers $(23,24)$. In 2009, Wagar et al. studied the humoral and cellular immune memory response in a few individuals who were infected and/or vaccinated (25). It should be noted that even though the serum $\mathrm{Ab}$ response to influenza infec- 
A

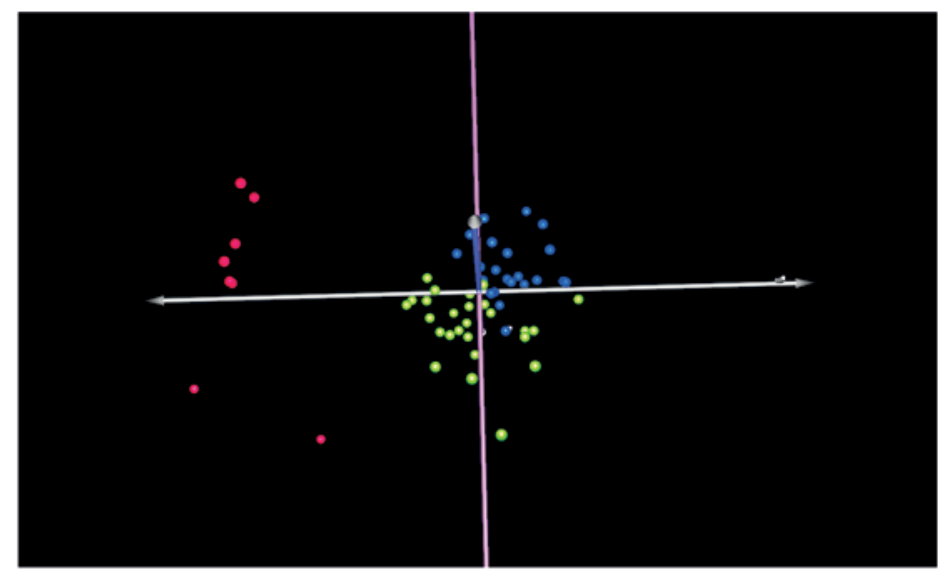

B
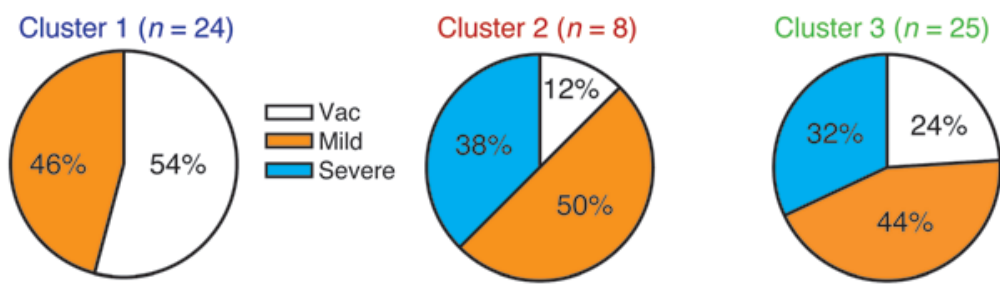

C

HI titers

\section{Figure 3}

Differential and similar mobilization of immune responses 1 year after $\mathrm{A}(\mathrm{H} 1 \mathrm{~N} 1)$ pdm09 vaccination or infection. (A) PCA of the immune responses showed that the 57 subjects with complete data could be segregated on the basis of $\log _{10}$ influenza $A(\mathrm{H} 1 \mathrm{~N} 1)$ pdm09-specific responses into 3 clusters: cluster 1 ( $n=24$; blue), cluster 2 ( $n=8$; red), and cluster 3 ( $n=25$; green). (B) The percentages of vaccinated subjects (white), mildly ill subjects (orange), and severely ill subjects (blue) for each cluster are summarized in the pie charts. (C) The radar chart presents the upper and lower $95 \%$ Cls of the means of each assay as indicated in linear scale, for cluster 1 (blue), cluster 2 (red), and cluster 3 (green). Statistical analyses used the Mann-Whitney $U$ test, and statistical significance is indicated. ${ }^{* *} P<0.01,{ }^{* * *} P<0.001$, ${ }^{* * * *} P<0.0001$.

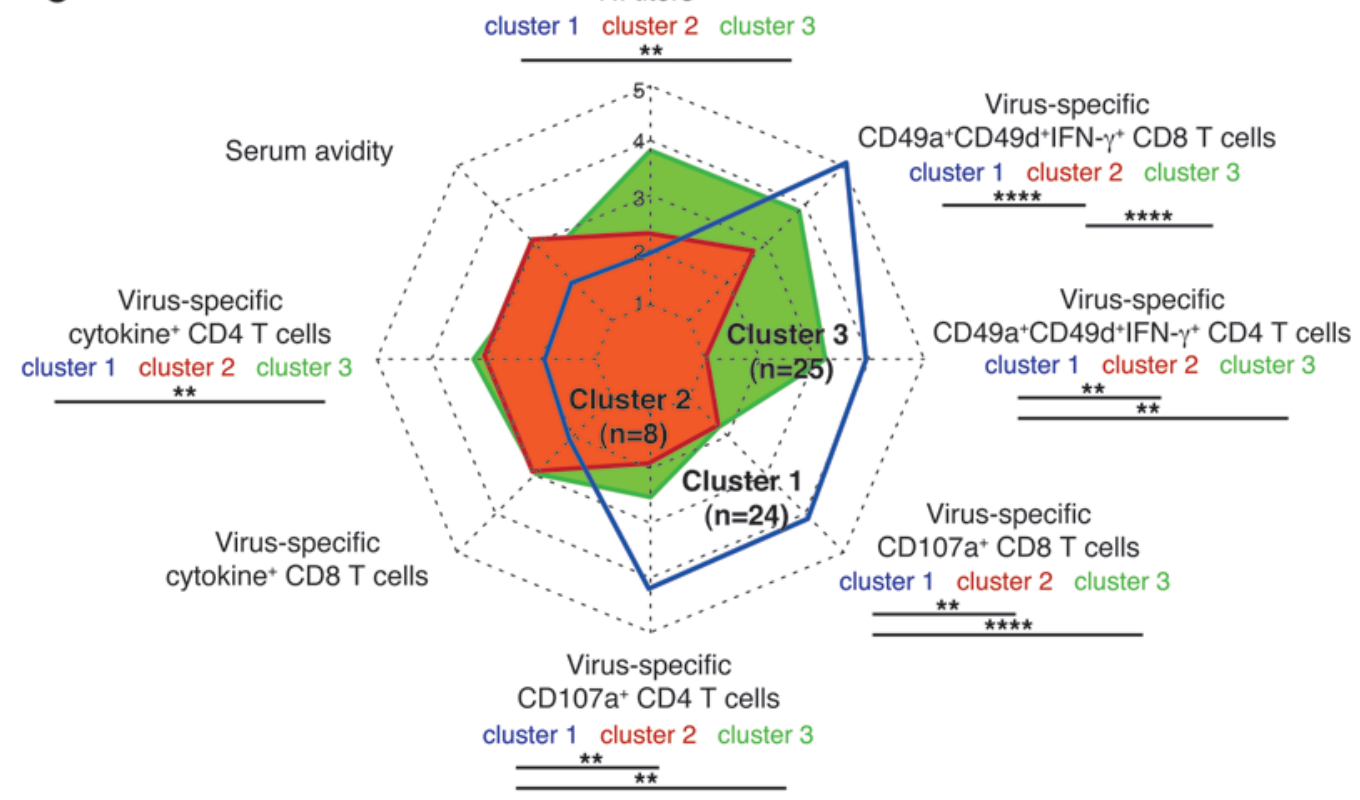

tion or vaccination was found to be ephemeral, this study failed to take initial disease severity into account in assessing the persistence of immune memory.

Our work, we believe, is unique in its comparison of the immune memory signature in individuals after vaccination, mild illness, and severe illness. Most of the parameters tested have been considered to be correlates or cocorrelates of protection against influenza viruses. Thus, the strength of our finding is that we considered numerous parameters in the persistence of correlates of protection after vaccination and infection. Recent additions to the literature offer relevant evidence about immune cell responses and influenza illness. Sridhar et al. (26) established that the high frequencies of
IFN- $\gamma^{+}$IL2- $\mathrm{CD}^{+}$cells and lung-homing and cytotoxic potential are strongly correlated with less severe illness. They proposed that, in the absence of crossreactive neutralizing Abs, these protective immune correlates might be used as "a guide for universal influenza vaccine development." This is consistent with our findings; both homing and the cytotoxic potential of $\mathrm{CD}^{+}$memory cells are features of both past vaccination and mild infection.

In addition, Wilkinson et al. (27) conducted an influenza infection study in healthy volunteers with no detectable Abs to the challenge viruses, $\mathrm{H} 3 \mathrm{~N} 2$ or $\mathrm{H} 1 \mathrm{~N} 1$. They showed that preexisting $\mathrm{CD}^{+}$ $\mathrm{T}$ cells (with evidence of cytotoxic activity), but not $\mathrm{CD}^{+} \mathrm{T}$ cells, were associated with low virus shedding and less severe illness. 
Our work compares 3 groups of individuals who first encountered this antigen in 3 different sets of circumstances: individuals who received an adjuvanted influenza vaccine, those who had mild infections, and those with severe infections. A year after this first antigen encounter, their immune memories are considered to have regained the resting state. However, this memory's persistence, nature, and intensity depend on the context of the antigenic stimulation (antigen dose, inflammatory reaction, and durability of antigenic exposure) $(20,28)$. One may question whether antiviral treatment decreased antigenic stimulation through its impact on viral shedding. All subjects except 1 with severe influenza received antiviral treatment at a median of 7 days (range: 3-11 days) after the onset of symptoms and thus were surely exposed to a high antigen dose before first treatment intake. In mild infection, viral shedding peaks on average 2 days after infection, that is, fewer than 3 hours after symptoms start (29). Given that the treatment is usually first administered between 12 and 48 hours after the onset of symptoms, here again, total viral shedding was at most only slightly modified by antiviral treatment. Thus, it is unlikely that antiviral treatments would affect the immune outcomes in these conditions.

At 1 year after pandemic, the serum Ab levels (geometric mean HI titers: 95) were considered seroprotective in $76 \%$ of those who were severely ill compared with those (geometric mean HI titers: 50 ) in $50 \%$ of the mildly ill individuals and $56 \%$ of those who were vaccinated. We also found high frequencies of polyfunctional antigen-specific $\mathrm{CD}^{+} \mathrm{T}$ cells producing multiple cytokines at 1 year after severe infections and lower frequencies after a mild infection or vaccination; these subjects had predominantly IFN- $\gamma^{+}-$ producing $\mathrm{CD}^{+} \mathrm{T}$ cells. We also found that the number of influenza A(H1N1)pdm09-specific T cells expressing degranulation markers (CD107a) in vaccinated individuals was highly variable, but that this population was not found at the memory phase in infected individuals. Of note, mouse $\mathrm{CD}^{+}{ }^{+}$effectors confer protection from lethal influenza through perforin-mediated cytotoxicity (30).

Because infection and vaccination are different routes of immunization that can dictate antigen imprinting and $\mathrm{T}$ cell homing (31-33), we also tested a mucosa-homing marker on T cells (19). A high level of both antigen-specific $\mathrm{CD}^{+}$and $\mathrm{CD}^{+} \mathrm{T}$ cells expressing CD $49 \mathrm{a}^{+} \mathrm{CD} 49 \mathrm{~d}^{+}$markers was observed in both mildly ill and vaccinated individuals a year after immunization, while the level was low in those who had had severe infections. One limitation of our study is that our overall picture of immunologic memory is that of the blood; it does not reflect tissue-associated immunity (upper airways). The low levels of mucosa-homing $\mathrm{T}$ cells at 1 year after severe infection might also reflect the sequestration (34) or exhaustion of these cells in the lungs during the initial infection.

In our quest for memory signatures of severe and mild infections and vaccination, we performed a PCA. The PCA enabled us to take the similarities between subjects into account to optimize the robustness of our information while preserving a portion of the variation of the initial data set. Interestingly, we found 3 clusters of subjects: the first cluster consisted of both vaccinated and mildly ill subjects and was characterized by a higher intensity of mucosal-homing and degranulation markers on influenza-specific T cells. However, clusters 2 and 3 underlined some similarities at the memory phase between severely and mildly ill individuals. Our results are the first, to our knowledge, to show that the initial context of immunization (severe or mild infection or vaccination) dictates the magnitude and quality of the immunologic memory, producing a distinct signature of severe infection, while mildly ill and vaccinated subjects share a different signature. The contribution of age difference was evaluated according to these 3 clusters of immune profiles. Individuals in cluster 3 were the youngest; this cluster also included the largest number of mildly ill subjects, who were younger that the others in our study (data not shown).

It might be argued that preexisting immunity, which is agedependent, thus influenced the severity of the influenza illness. Unfortunately, data on preexisting immune responses are not available in our study groups: what we have is a snapshot of the immune memory against influenza. We recently published an analysis of immune responses before and after vaccination at day 21 and at memory phase month 4 (21). We found that the higher the baseline (of preexisting flu-specific immune responses) was, the lower the amplification of responses was at 3 weeks after immunization $(\Delta$ between baseline and 3 weeks for effector immune responses). We did find significant differences in the intensity of the cellular and humoral responses at the memory phase (month 4) between the low- and high-baseline groups; this suggest that baseline immune responses affect the amplitude of immune memory (21).

A global description distinguishing immune responses to vaccine and to infection is a cost-effective albeit laborious task. The difficulty arises in part from the multiplicity of phenotypes and functions that can be studied for each immune compartment. However, comparing the immune memory after vaccination and infection is essential for designing novel vaccination strategies.

\section{Methods}

Study design. This study included 50 vaccinated volunteers, 48 patients with mild to moderate $\mathrm{A}(\mathrm{H} 1 \mathrm{~N} 1) \mathrm{pdm} 09$ pandemic virus influenza infection, and 13 with severe infection. Subjects had to meet the following eligibility criteria: age from 18 to 65 years, clinical examination and interview for medical history, documented history of influenza vaccination, no psychological, familial, sociological or geographic condition that might impede compliance with the study protocol, and written informed consent. Further exclusion criteria were pregnancy or any acute or chronic illness or local or systemic immunosuppressive treatments that might interfere with the study protocol. Vaccinated volunteers and infected patients from several French hospitals were enrolled in this protocol from September 16 through November 22, 2010.

All vaccinated subjects were recruited from among hospital health care staff and received the $\mathrm{A}(\mathrm{H} 1 \mathrm{~N} 1) \mathrm{pdm} 09$ adjuvanted vaccine (Pandemrix, GlaxoSmithKline) by the intramuscular route.

Positive RT-PCR for influenza A(H1N1)pdm09 virus during winter 2009 verified the pandemic influenza of all infected subjects (mild and severe), and none received any influenza $\mathrm{A}(\mathrm{H} 1 \mathrm{~N} 1) \mathrm{pdm} 09$ vaccination. So-called mildly ill patients reported an uncomplicated flu syndrome during the 2009-2010 seasonal flu period. All were recruited from the GROG network or from dedicated pandemic consultation center registries. During this period, severely ill patients were diagnosed with acute respiratory distress syndrome (ARDS) (defined by the American-European Consensus Conference on ARDS) (35) caused by A(H1N1)pdm09 virus infection. All were recruited from the Réseau Européen de Ventilation Artificielle (REVA) registry (36). Table 1 summarizes the characteristics of all 3 groups of subjects.

At 10 to 12 months (autumn 2010) after their influenza A(H1N1)pdm09 vaccination or infection, subjects provided blood samples for immunologic analyses. PBMCs were isolated on Ficoll gradients (Eurobio). Sera and cells were frozen before these analyses. The investigators performing the immunologic analyses were masked to participant vaccination or infection status and remained so until the end of the study. 
Table 1

Demographic characteristics of the subjects

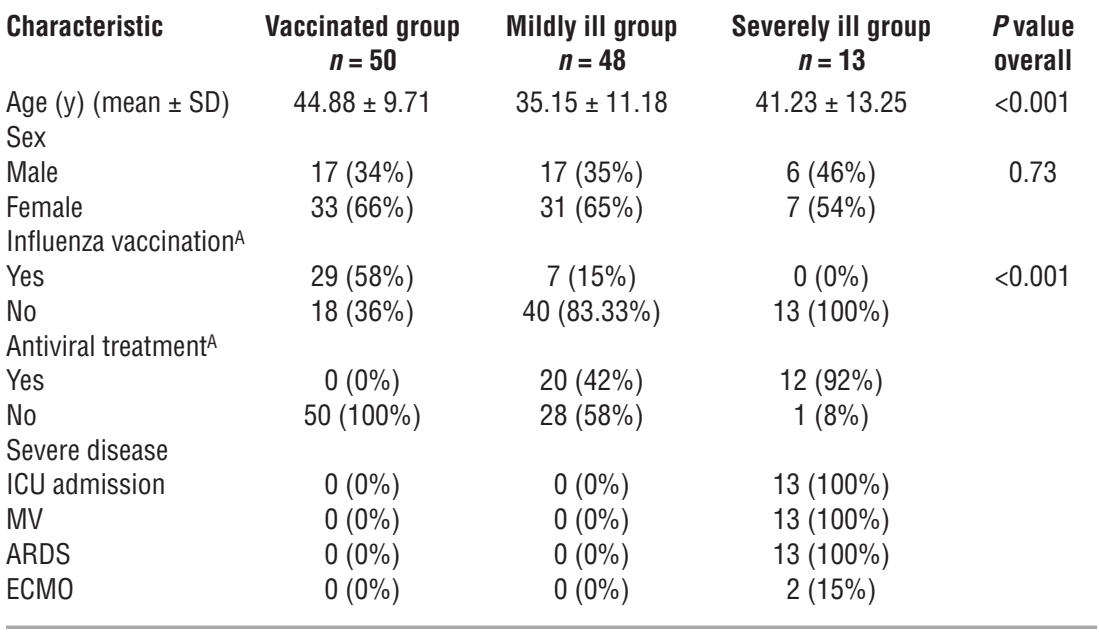

ICU, intensive care unit; MV, mechanical ventilation; ECMO, extracorporeal membrane oxygenation. ADuring 2009-2010 influenza season. Statistical analyses used Fisher's exact test for qualitative and Kruskal-Wallis test for quantitative analyses. Statistical significance was set at $P<0.5$.

live events, according to forward- and sidescatter parameters, were accumulated and analyzed for Boolean combination gating with FlowJo software (Tree Star Inc.). Background cytokine responses detected in negative controls were subtracted from those detected in stimulated samples for every specific combination. The percentage of influenza $\mathrm{A}(\mathrm{H} 1 \mathrm{~N} 1)$ pdm09 virus-specific $\mathrm{CD}^{+}$or $\mathrm{CD}^{+} \mathrm{T}$ cells expresses the sum of the 7 different cytokine combinations. Differentiation and homing molecule analysis were done with virus-stimulated PBMCs.

Radar charts and PCA. The radar charts were designed with $\mathrm{R}$, a free software environment for statistical computing and graphics (http:// www.r-project.org/). The PCA and hierarchical clustering (HCL) analysis used the data of influenza virus immune responses. Data for all 8 parameters were available for 57 subjects. To stratify the population and visualize the clusters, we used a part of the TM4 software suite, the Multi Experiment Viewer, $\mathrm{MeV}$ (39). $\log _{10}$ values were normalized with $\mathrm{MeV}$ 4.7.4

Hemagglutination inhibition assay. Serum Abs against nonadjuvanted $\mathrm{A}(\mathrm{H} 1 \mathrm{~N} 1) \mathrm{pdm} 09$ influenza vaccine (Panenza; Sanofi Pasteur) were measured by a microtiter HI assay modified from Kendal et al. (37). Briefly, after treatment by receptor-destroying enzyme (RDE), serial 2-fold dilutions of serum (from 1:10) were tested against 4 HA units of antigen, on human $\mathrm{O} \mathrm{Rh}^{-}$red blood cells. The HI titers were defined as the reciprocal of the highest serum dilution that completely inhibited hemagglutination. Serum avidity assay. Serum avidity of anti-HA Abs was evaluated by ELISA (38). Serum samples from donors were incubated with recombinant $\mathrm{HA}$ from A/California/07/2009(H1N1) influenza virus (Protein Sciences) coated on ELISA plates at a dilution equivalent to $2 \mu \mathrm{g} / \mathrm{ml}$ of $\mathrm{HA}$, in the presence of serial dilutions of sodium thiocyanate (NaSCN) (Sigma-Aldrich). HRPconjugated mouse anti-human IgG (Southern Biotech) was incubated before revelation with o-phenylenediamine peroxidase substrate buffer. The reaction was stopped with $2 \mathrm{~N} \mathrm{HCl}$, and optical density was read with a GENios microplate reader at $492 \mathrm{~nm}$ with Xfluor4 software. Serum avidity was defined as the concentration of $\mathrm{NaSCN}$ required to induce a $50 \%$ inhibition of Ab binding.

Cell stimulation and intracellular cytokine staining (ICS). Frozen PBL were available for further analysis of influenza $\mathrm{A}(\mathrm{H} 1 \mathrm{~N} 1) \mathrm{pdm} 09$ virus-specific $\mathrm{T}$ cells. Cells were infected for 1 hour at $37^{\circ} \mathrm{C}$ with or without (negative control) live pandemic influenza A/California/07/2009(H1N1) virus in RPMI 1640 without FCS and washed before overnight incubation. Brefeldin A and monensin (Sigma-Aldrich) were added for the last 14 hours in the presence of CD107a-PE-Cy5 mAb (BD Biosciences). Cells were washed and stained with CD3-AmCyan, CD4-Pacific blue, and CD8-APC-H7, (Beckman Coulter) mAbs. The BD Cytofix/Cytoperm kit was used to permeabilize cells, in accordance with the manufacturer's instructions, before staining for the following intracellular markers: IL-2-FITC, IFN- $\gamma$-Alexa Fluor 700, and TNF- $\alpha-\mathrm{PE}-\mathrm{Cy} 7 \mathrm{mAbs}$ (BD Biosciences). Two different $\mathrm{Ab}$ panels were used as follows: CD3-AmCyan/CD4-Pacific blue/CD8-APCH7/CD27-PE/CD45RA-ECD/CD107a-PE-Cy5/IL-2-FITC/IFN- $\gamma$-Alexa Fluor $700 /$ TNF- $\alpha$-PE-Cy7 or CD3-AmCyan/CD4-Pacific blue/CD8-APCH7/CCR5-FITC/CXCR3-PE-Cy7/CD49a-PE/CD49d-PE-Cy5/IFN- $\gamma$-Alexa Fluor 700. Flow cytometry analyses were performed with an LSRII flow cytometer (BD Biosciences Immunocytometry Systems). At least $1 \times 10^{6}$ software. The best division for the initial population was obtained for 3 clusters (inflexion point of the Figure of Merit; ref. 40). To cluster the data, e.g., to stratify the initial population, we ran the k-means clustering algorithm for 3 clusters and performed HCL analysis on the items in each cluster thus created (41). HCL analysis was done with complete linkage and Pearson correlation. Each cluster was then colored separately. A PCA was run and used to attribute the overall variability in the data to a reduced set of variables, that is, the principal components. We used the first 3 principal components to map each element into a $3 \mathrm{D}$ viewer. This mathematical operation reduces the dimensionality of the data and is a simple nonparametric method of extracting relevant information from a confusing/ complex data set $(42,43)$. This analysis also helped to define the relevant signatures associated with infection or vaccination at the memory phase.

Statistics. We used the Mann-Whitney test to compare quantitative variables across groups, Kruskal-Wallis test for quantitative variables and Fisher's exact test to compare 3 qualitative variables. Nonparametric Spearman correlations were used for tests of 2 quantitative variables. Statistical significance was set at $P<0.05$. All statistical analyses were performed with SPSS statistical software 17.0, SAS 9.2 (SAS Institute Inc.), and Prism 5.0 or Microsoft Excel for Mac OS X was used for data handling and graphic representation.

Study approval. The trial was conducted in accordance with the latest amended version of the Declaration of Helsinki, Good Clinical Practice (GCP), and International Conference on Harmonisation (ICH) regulatory guidelines. The study protocol, its first amendment, and the informed consent and patient information forms were reviewed and approved by the independent Ethics Committee of Pitié-Salpêtrière Hospital (Approved by CPP Ile de France $3 \mathrm{~N}^{\circ}$ ID-RCB: 2010-A00631-38). Written informed consent was obtained from each volunteer before study entry.

\section{Acknowledgments}

This study was supported by a grant from the Programme de Recherche, $\mathrm{A}(\mathrm{H} 1 \mathrm{~N} 1)$ coordinated by the Institut de Microbiologie et Maladies Infectieuses (IMMI, France). The study sponsor was not involved in the study design, monitoring, or in the data analysis or interpretation. Immunomonitoring analysis was funded by the 
Fondation pour la Recherche Médicale. B. Combadière is the recipient of an INSERM-Interface AP/HP award, and the Fondation pour la Recherche Médicale "Equipe.” We thank members of the REVA network and the GROG network and the dedicated pandemic consultation centers for selection and recruitment of the patients, and members of Memo-Flu-ARDS study group: Christian Dualé (INSERM CIC 501, Centre Hospitalier Universitaire, ClermontFerrand, France), Jean-Paul Bernard (Assistance Publique-Hôpitaux de Marseille, Marseille, France), René Robert (CHU de Poitiers, Service de Réanimation Médicale, Poitiers, France), Fabrice Lainé (CIC P-0203 de Rennes, Centre Hospitalier Universitaire de Rennes, Rennes, France), Louis Bernard (Médecine Interne et Maladies Infectieuses, Centre Hospitalier Régional Universitaire de Tours, Tours, France), Francois Bricaire (Infectious and Tropical Diseases Unit, Hôpital Pitié-Salpêtrière, AP/HP), Alain Combes (Service de Réanimation Médicale, Institut de Cardiologie, Groupe Hospitalier Pitié-Salpêtrière, AP/HP, Paris, France), Xavier Duval (CIC P-007, CHU Bichat-Claude Bernard, Paris, France), Stéphane Jaureguiberry
(Unité des Maladies Infectieuses et Tropicales, Hôpital PitiéSalpêtrière, AP/HP), Christian Perronne (Unité des Maladies Infectieuses et Tropicales, Hôpital Raymond Poincaré, AP/HP, Garches, France), Christophe Rapp (Service des Maladies Infectieuses et Tropicales, Hôpital d'Instruction des Armées Bégin, Saint Mandé, France), Dominique Salmon-Céron (Pôle de Médecine - Unité de Pathologie Infectieuse, Hôpital Cochin, AP/HP, Paris, France), and Daniel Vittecoq (Service des Maladies Infectieuses et Tropicales, Hôpital de Bicêtre, AP/HP, Le Kremlin-Bicêtre, Paris, France).

Received for publication December 3, 2013, and accepted in revised form April 10, 2014.

Address correspondence to: Behazine Combadiere, INSERM UMR 1135, Centre d'Immunologie et des Maladies Infectieuses Paris (Cimi-Paris), 91 Boulevard de l'Hôpital, 75013 Paris, France. Phone: 33.1.4077988; Fax: 33.1.40779734; E-mail: behazine. combadiere@upmc.fr.
1. Dawood FS, et al. Emergence of a novel swine-origin influenza A (H1N1) virus in humans. $N$ Engl J Med. 2009;360(25):2605-2615.

2. Webster RG, Laver WG, Air GM, Schild GC. Molecular mechanisms of variation in influenza viruses. Nature. 1982;296(5853):115-121.

3. Garten RJ, et al. Antigenic and genetic characteristics of swine-origin $2009 \mathrm{~A}(\mathrm{H} 1 \mathrm{~N} 1)$ influenza viruses circulating in humans. Science. 2009; 325(5937):197-201.

4. Smith DJ, Forrest S, Ackley DH, Perelson AS. Variable efficacy of repeated annual influenza vaccination. Proc Natl Acad Sci U S A. 1999;96(24):14001-14006.

5. Assarsson E, et al. Immunomic analysis of the repertoire of T-cell specificities for influenza A virus in humans. J Virol. 2008;82(24):12241-12251.

6. Greenbaum JA, et al. Pre-existing immunity against swine-origin H1N1 influenza viruses in the general human population. Proc Natl Acad Sci U S A. 2009;106(48):20365-20370.

7. McMichael AJ, Gotch FM, Noble GR, Beare PA. Cytotoxic T-cell immunity to influenza. $N$ Engl J Med. 1983;309(1):13-17.

8. Chowell G, et al. Severe respiratory disease concurrent with the circulation of H1N1 influenza. NEngl JMed. 2009;361(7):674-679.

9. Pelat C, et al. Field effectiveness of pandemic and 2009-2010 seasonal vaccines against 2009-2010 $\mathrm{A}(\mathrm{H} 1 \mathrm{~N} 1)$ influenza: estimations from surveillance data in France. PLoS One. 2011;6(5):e19621.

10. Ploin D, et al. Complications and factors associated with severity of influenza in hospitalized children and adults during the pandemic wave of $\mathrm{A}(\mathrm{H} 1 \mathrm{~N} 1)$ pdm2009 infections - the Fluco French cohort. J Clin Virol. 2013;58(1):114-119.

11. Zarychanski R, et al. Correlates of severe disease in patients with 2009 pandemic influenza (H1N1) virus infection. CMAJ. 2010;182(3):257-264.

12. Monsalvo AC, et al. Severe pandemic $2009 \mathrm{H} 1 \mathrm{~N} 1$ influenza disease due to pathogenic immune complexes. Nat Med. 2011;17(2):195-199.

13. To KK, et al. Delayed clearance of viral load and marked cytokine activation in severe cases of pandemic H1N1 2009 influenza virus infection. Clin Infect Dis. 2010;50(6):850-859.

14. To KK, et al. High titer and avidity of nonneutralizing antibodies against influenza vaccine antigen are associated with severe influenza. Clin Vaccine Immunol. 2012;19(7):1012-1018.

15. Hobson D, Curry RL, Beare AS, Ward-Gardner A. The role of serum haemagglutination-inhibiting antibody in protection against challenge infec- tion with influenza A2 and B viruses. J Hyg (Lond). 1972;70(4):767-777.

16. Gulati U, Kumari K, Wu W, Keitel WA, Air GM. Amount and avidity of serum antibodies against native glycoproteins and denatured virus after repeated influenza whole-virus vaccination. Vaccine. 2005;23(11):1414-1425.

17. Seder RA, Darrah PA, Roederer M. T-cell quality in memory and protection: implications for vaccine design. Nat Rev Immunol. 2008;8(4):247-258.

18. McMichael AJ, Askonas BA. Influenza virusspecific cytotoxic $T$ cells in man; induction and properties of the cytotoxic cell. Eur J Immunol. 1978;8(10):705-711.

19. Richter $\mathrm{M}$, et al. Collagen distribution and expression of collagen-binding $\alpha 1 \beta 1$ (VLA- 1 ) and $\alpha 2 \beta 1$ (VLA-2) integrins on CD4 and CD8 T cells during influenza infection. JImmunol. 2007;178(7):4506-4516.

20. Ahmed R, Gray D. Immunological memory and protective immunity: understanding their relation. Science. 1996;272(5258):54-60.

21 . Bonduelle $\mathrm{O}$, et al. Longitudinal and integrative biomodeling of effector and memory immune compartments after inactivated influenza vaccination. J Immunol. 2013;191(2):623-631.

22. Raychaudhuri S, Stuart JM, Altman RB. Principal components analysis to summarize microarray experiments: application to sporulation time series. Pac Symp Biocomput. 2000;455-466.

23 . Hancock K, et al. Cross-reactive antibody responses to the 2009 pandemic H1N1 influenza virus. N Engl J Med. 2009;361(20):1945-1952.

24. Itoh $Y$, et al. In vitro and in vivo characterization of new swine-origin $\mathrm{H} 1 \mathrm{~N} 1$ influenza viruses. Nature. 2009;460(7258):1021-1025.

25. Wagar LE, et al. Humoral and cell-mediated immunity to pandemic H1N1 influenza in a Canadian cohort one year post-pandemic: implications for vaccination. PLoS One. 2011;6(11):e28063.

26 . Sridhar S, et al. Cellular immune correlates of protection against symptomatic pandemic influenza. Nat Med. 2013;19(10):1305-1312.

27. Wilkinson TM, et al. Preexisting influenza-specific CD4(+) T cells correlate with disease protection against influenza challenge in humans. Nat Med. 2012;18(2):274-280.

28. Marois I, Cloutier A, Garneau E, Richter MV. Initial infectious dose dictates the innate, adaptive, and memory responses to influenza in the respiratory tract. J Leukoc Biol. 2012;92(1):107-121.

29. Canini L, Carrat F. Population modeling of influenza A/H1N1 virus kinetics and symptom dynam- ics. J Virol. 2011;85(6):2764-2770.

30. Brown DM, Dilzer AM, Meents DL, Swain SL. CD4 $\mathrm{T}$ cell-mediated protection from lethal influenza: perforin and antibody-mediated mechanisms give a one-two punch. J Immunol. 2006;177(5):2888-2898.

31. Estcourt MJ, Letourneau S, McMichael AJ, Hanke T. Vaccine route, dose and type of delivery vector determine patterns of primary $\mathrm{CD}^{+} \mathrm{T}$ cell responses. Eur J Immunol. 2005;35(9):2532-2540.

32. Abadie V, Bonduelle O, Duffy D, Parizot C, Verrier $\mathrm{B}$, Combadiere B. Original encounter with antigen determines antigen-presenting cell imprinting of the quality of the immune response in mice. PLoS One. 2009;4(12):e8159.

33. Combadiere B, Liard C. Transcutaneous and intradermal vaccination. Hum Vaccin. 2011;7(8):811-827.

34. de Bree GJ, van Leeuwen EM, Out TA, Jansen HM, Jonkers RE, van Lier RA. Selective accumulation of differentiated $\mathrm{CD}^{+} \mathrm{T}$ cells specific for respiratory viruses in the human lung.J Exp Med. 2005;202(10):1433-1442.

35. Bernard GR, et al. The American-European Consensus Conference on ARDS. Definitions, mechanisms, relevant outcomes, and clinical trial coordination. Am J Respir Crit Care Med. 1994;149(3 pt 1):818-824.

36. Luyt CE, et al. Long-term outcomes of pandemic 2009 influenza A(H1N1)-associated severe ARDS. Chest. 2012;142(3):583-592.

37. Kendal AP, Cate TR. Increased sensitivity and reduced specificity of hemagglutination inhibition tests with ether-treated influenza B/Singapore/222/79. J Clin Microbiol. 1983;18(4):930-934.

38. Dimitrov JD, Lacroix-Desmazes S, Kaveri SV. Important parameters for evaluation of antibody avidity by immunosorbent assay. Anal Biochem. 2011; 418(1):149-151.

39. Saeed AI, et al. TM4: a free, open-source system for microarray data management and analysis. Biotechniques. 2003;34(2):374-378.

40. Yeung KY, Haynor DR, Ruzzo WL. Validating clustering for gene expression data. Bioinformatics. 2001; 17(4):309-318.

41. Soukas A, Cohen P, Socci ND, Friedman JM. Leptin-specific patterns of gene expression in white adipose tissue. Genes Dev. 2000;14(8):963-980.

42. Daffertshofer A, Lamoth CJ, Meijer OG, Beek PJ. PCA in studying coordination and variability: a tutorial. Clin Biomech (Bristol, Avon). 2004;19(4):415-428.

43. Roden JC, King BW, Trout D, Mortazavi A, Wold BJ, Hart CE. Mining gene expression data by interpreting principal components. BMC Bioinformatics. 2006;7:194. 